\title{
A Novel Superhard Tetragonal Carbon Mononitride
}

\author{
Meiguang Zhang, ${ }^{* \dagger}$ Qun Wei, ${ }^{*}+$ Haiyan Yan, ${ }^{\dagger} \S$ Yaru Zhao, ${ }^{\dagger}$ and Hui Wang" \\ ${ }^{\dagger}$ Department of Physics and Information Technology, Shaanxi Key Laboratory for Phytochemistry, Baoji University of Arts and \\ Sciences, Baoji, Shaanxi 721016, P. R. China \\ ${ }^{\ddagger}$ School of Physics and Optoelectronic Engineering, Xidian University, Xi'an, Shaanxi 710071, P. R. China \\ ${ }^{\S}$ College of Chemistry and Chemical Engineering, Baoji University of Arts and Sciences, Baoji, Shaanxi 721013, P. R. China \\ "National Laboratory of Superhard Materials, Jilin University, Changchun, Jilin 130012, P. R. China
}

ABSTRACT: We introduced a novel carbon mononitride within tetragonal $\mathrm{P} 4_{2} / \mathrm{m}$ symmetry motivated by experimental synthesis and theoretical predictions. Phonon dispersion and formation enthalpy calculations suggest that $P 4_{2} / m-\mathrm{CN}$ is dynamically stable at ambient conditions and can be synthesized at readily attainable pressures. The crystal orientation dependences of the Young's and shear moduli have been systematically studied for this $P 4_{2} / m$ phase. Further mechanical calculations suggested that the $P 4_{2} / m$-CN is ultracompressible and superhard. The ideal tensile and shear strength at large strains of $P 4_{2} / m$-CN are also examined. The results suggest that the weakest tensile strength is along the [100] direction, and the atomic deformation mechanism of $P 4_{2} / m-C N$ along the weakest shear strength direction (100) [010] is discussed.

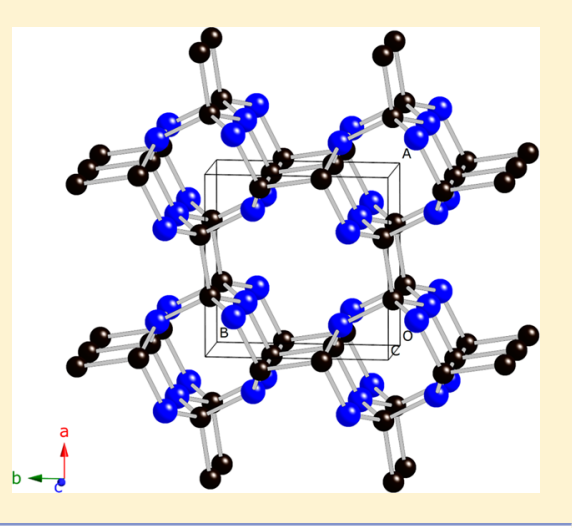

\section{INTRODUCTION}

The search for intrinsic superhard materials is inspired by the technological need for robust and chemically stable materials in such applications as cutting tools and wear resistant coatings. Carbon nitrides have attracted much attention for the belief that relatively short bond lengths and low bond ionicity in these materials make them primary candidates for low-compressibility or superhard materials. ${ }^{1}$ The prediction of hexagonal $\beta-\mathrm{C}_{3} \mathrm{~N}_{4}{ }^{2,3}$ with extraordinary hardness has led to a great deal of experimental and theoretical works searching for new $\mathrm{CN}$ materials with novel properties. ${ }^{4-13}$ On the experimental side, various carbon-nitrogen rich compounds such as melamine, cyanamide, and other related triazine-based compounds ${ }^{14-16}$ have been used as precursors to synthesize carbon nitride phases by using various techniques. ${ }^{15,17-19}$ However, in addition to the limited quality of samples in actual experiments, most experimental results have resolutions too low to accurately identify crystal structures and internal atomic arrangement because of the similar and small atomic masses and rich chemical binding of $\mathrm{C}$ and $\mathrm{N}$ atoms. On the theoretical side, several theoretical approaches ${ }^{4,9,12,20-23}$ have been applied to predict and design new carbon nitrides, among which stateof-the-art structure prediction methods ${ }^{12,23}$ based on different algorithms are the most prevalent strategies to explore and predict new material structures. One of the most successful examples is that the predicted carbon allotrope, $M$-carbon, ${ }^{24}$ was finally confirmed by experiments to be cold-compressed graphite. $^{25,26}$ Therefore, theoretical calculations can provide a powerful tool for further exploration of new structures (including atomistic structure, stability, related physical properties, etc.) to complement experiments.
Our present work on the structure of carbon mononitride is motivated by the previous experimental synthesis of a tetragonal $\mathrm{C}-\mathrm{N}$ compound. ${ }^{5}$ This new $\mathrm{C}-\mathrm{N}$ compound was first experimentally synthesized in the carbon-nitrogen films on $\mathrm{Ni}(100)$ substrates by Guo et al., and the energy dispersive $\mathrm{X}$-ray analysis gives a relative $\mathrm{N} / \mathrm{C}$ raio of $0.8-1.0{ }^{5}$ However, the stoichiometry and atomic arrangement of the $\mathrm{C}-\mathrm{N}$ phase have not yet been determined experimentally. Extensive theoretical studies are thus carried out for $\mathrm{CN}$ structure with a 1:1 stoichiometry, especially on the fundamental crystal structures. Using a self-consistent charge density functional tight-binding method, Kim et al. ${ }^{7,8}$ investigated the structural stability of two tetragonal phases (tetragonal rocksalt phase and the $\beta$-tin phase) for $\mathrm{CN}$ and examined the variation of equilibrium volume and the bulk modulus with changing nitrogen content. Recently, Hart et al. ${ }^{9}$ and Wang et al. ${ }^{23,27}$ have systematically investigated the structural and mechanical properties of $\mathrm{CN}$ phase within GaSe, $\beta$-InS, rocksalt, $b c t-4, b c c$, $c g-\mathrm{CN}$, and Pnnm structures. Among these candidates, the $\mathrm{sp}^{3}$ hybridized CN phase with Pnnm symmetry is found to be the most preferential structure with claimed hardness of 62.3 GPa. These important pioneering works give us broadened views into the essence of atomic binding in solids while advancing our understanding of the structural stability of this novel CN phase. Although so many different kinds of structures are proposed, the quest for a new energetically stable or metastable $\mathrm{CN}$ phase is one of the hot topics in condensed matter physics. In this

Received: September 12, 2013

Revised: January 20, 2014

Published: January 24, 2014 
work, we applied a recently developed crystal structure analysis by partical swarm optimization (CALYPSO) ${ }^{28}$ algorithm to extensively explore the most energetically stable $\mathrm{CN}$ phase at ambient pressure, unbiased by prior known structural information. This method has been successfully applied to several structures that have been confirmed by independent experiments. ${ }^{29-31}$ Indeed, a novel tetragonal $\mathrm{P}_{2} / \mathrm{m}$-CN built up by strong covalent $\mathrm{C}-\mathrm{N}$ bonds and $\mathrm{C}-\mathrm{C}$ bonds is uncovered, which is energetically much superior to the best previous reported candidate Pnnm-CN. First-principles calculations were then performed to characterize the structural, thermodynamic, mechanical, and electronic properties of this novel tetragonal $\mathrm{CN}$ phase.

\section{COMPUTATIONAL METHODS}

The crystal structure prediction is based on a global minimization of energy surfaces merging $a b$ initio total-energy calculations as implemented in CALYPSO code. $^{32} \mathrm{We}$ performed variable cell structure searches containing 1-6 formula units (f.u.) in the simulation cell at 0 GPa. The underlying $\mathrm{ab}$ initio structural relaxations and electronic calculations were performed using the generalized gradient approximation as implemented in the VASP code. ${ }^{33,34}$ The frozen-core all-electron projector augmented wave method ${ }^{35}$ was adopted with $2 s^{2} 2 p^{2}$ and $2 s^{2} 2 p^{3}$ treated as valence electrons for $\mathrm{C}$ and $\mathrm{N}$, respectively. To reduce the errors, the cutoff energy for the expansion of the wave function into plane waves was set to $900 \mathrm{eV}$, and the Monkhorst-Pack grid $(10 \times 10 \times$ $20)^{36}$ was individually adjusted in reciprocal space to the size of each computational cell. Single-crystal constants were determined by applying an appropriate set of distortions ${ }^{37}$ with the distortion parameter varying between \pm 0.001 to the equilibrium lattice. The polycrystalline bulk modulus, shear modulus, Young's modulus, and Poisson's ratio were thus estimated by using the Voigt-Reuss-Hill approximation ${ }^{38}$ The quasistatic ideal strength and relaxed loading path in the various directions were calculated using a method ${ }^{39}$ described previously. The phonon calculations were carried out using a supercell approach as implemented in the PHONOPY code. ${ }^{40}$

\section{RESULTS AND DISCUSSION}

When the CALYPSO method for $\mathrm{CN}$ at ambient pressure is employed, our structure searches reveal the most stable structure to be a tetragonal $P 4_{2} / m$ phase, which contains 4 f.u. in a unit cell. Interestingly, the previous proposed Pnnm structure was also predicted as a metastable structure with respect to the $P 4_{2} / \mathrm{m}$ phase. The crystal structure of $P 4_{2} / \mathrm{m}-\mathrm{CN}$ is shown in Figure 1. This tetragonal structure adopts a fully $3 \mathrm{D}$ packing consisting of intriguing helical tunnels connected to each other by $\mathrm{C}-\mathrm{C}$ covalent bonds. Inspection reveals that all the $\mathrm{C}$ atoms are tetrahedrally bonded with three $\mathrm{N}$ atoms and one $\mathrm{C}$ atom and that each $\mathrm{N}$ atom forms three single $\mathrm{C}-\mathrm{N}$ covalent bonds with three $\mathrm{C}$ neighbors. As is known for carbon nitrides, four-coordinated carbon and three-coordinated nitrogen leads to high bulk modulus and large hardness values. ${ }^{12}$ At $0 \mathrm{GPa}$, the calculated lattice parameters of $P 4_{2} / \mathrm{m}$-CN are $a=b$ $=4.666 \AA$ and $c=2.373 \AA$ in a unit cell in which two nonequivalent atoms $\mathrm{C}$ and $\mathrm{N}$ occupy the Wyckoff $4 j(0.3323$, $0.9737,0.0)$ and $4 j(0.8136,0.7391,0.0)$ sites, respectively. In more detail, the $\mathrm{C}-\mathrm{C}$ bond length in the $\mathrm{P}_{2} / \mathrm{m}$ phase is 1.584 $\AA$ at $0 \mathrm{GPa}$, slightly longer than that in diamond (1.535 $\AA$ ) but shorter than that in Pnnm phase (1.606 ̊). In the helical

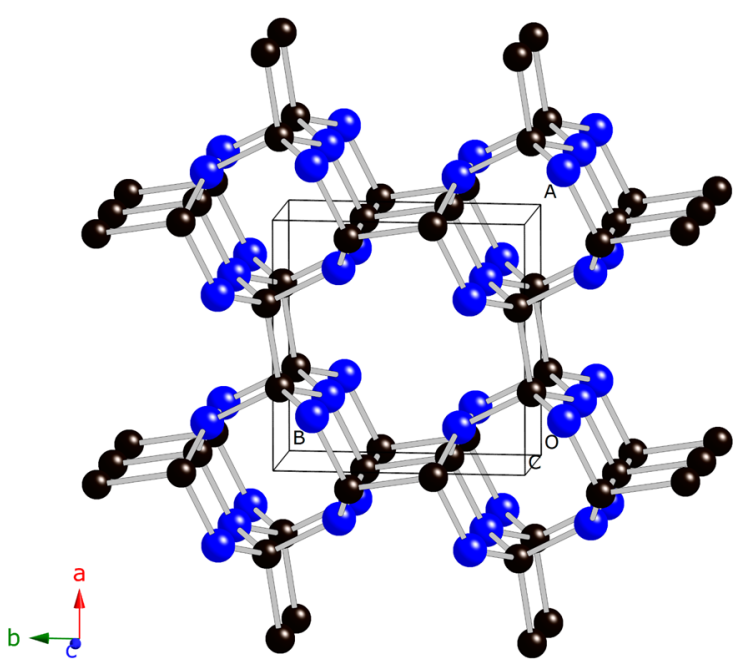

Figure 1. Crystal structure of $P 4_{2} / m$-CN. The blue and black spheres represent $\mathrm{N}$ and $\mathrm{C}$ atoms, respectively.

tunnels presented in Figure 1, the $\mathrm{C}-\mathrm{N}$ bond distances are calculated to be 1.441 and $1.504 \AA$, which are close to those in the Pnnm phase $(1.433 \text { and } 1.487 \AA)^{27}$ and $\beta-C_{3} \mathrm{~N}_{4}(1.442$, 1.444 , and $1.452 \AA){ }^{2}$ Our phonon calculations have verified that $P 4_{2} / m-C N$ is dynamically stable as evidenced by the absence of any imaginary frequency in the whole Brillouin zone at ambient pressure (see Figure 2c). Furthermore, the calculated zone-center phonon eigenvectors for $\mathrm{P}_{2} / \mathrm{m}-\mathrm{CN}$ were used to deduce the symmetry labels of the particular modes. According to group theory, 24 vibrational modes of $\mathrm{P}_{2} / \mathrm{m}$ at the zone-center have the irreducible representations $\Gamma_{P 4_{2} / m}=2\left(2 A_{\mathrm{g}}{ }^{R}+2 B_{\mathrm{g}}{ }^{R}+{ }^{1} E_{\mathrm{g}}{ }^{R}+{ }^{2} E_{\mathrm{g}}{ }^{R}+A_{\mathrm{u}}{ }^{I}+2^{1} E_{\mathrm{u}}{ }^{I}+2^{2} E_{\mathrm{u}}{ }^{I}\right)$. The Raman-active modes are labeled by the superscript $R$ and infrared-active ones by $I$. Therefore, further experimental work is expected to identify this $P 4_{2} / \mathrm{m}$ structure (zone-center phonons in Figure 2c) based on the calculated Raman and infrared frequencies.

Our computational approach is based on constant-pressure static quantum mechanical calculations at $T=0 \mathrm{~K}$; therefore, the relative stability of different phases of $\mathrm{CN}$ can be deduced from the pressure dependence of the enthalpy instead of the Gibbs free energy. To explore the thermodynamic stability for further experimental synthesis, the formation enthalpy of $\mathrm{P}_{2} /$ $m$-CN with respect to the separate phases is quantified by $\Delta H_{\mathrm{f}}$ $=H_{(\mathrm{CN})}-H_{(\mathrm{C})}-1 / 2 H_{\left(\mathrm{N}_{2}\right)}$; the graphite and solid-state molecular form of nitrogen ${ }^{41}$ are chosen as the reference phases. From Figure 2a, the formation enthalpies suggest that this $P 4_{2} / m$ phase is thermodynamically unstable at zero pressure because of its positive value. Like diamond, $c$-BN, and $\mathrm{C}_{3} \mathrm{~N}_{4}$, are all metastable compounds under atmospheric pressure. A thermodynamically stable phase is observed at 10.5 $\mathrm{GPa}$, which is close to that of the Pnnm phase (10.9 GPa) reported by Wang et al. ${ }^{27}$ Besides, the use of high temperature is a classical way to improve both diffusion processes and the reactivity of precursors for material synthesis of bulk forms or thin films. Studies of thermodynamic stability and physical properties of the $\mathrm{CN}$ compound under high temperature would also improve the understanding of the synthesis. However, these studies are beyond the scope of the current work. Furthermore, an enthalpy difference curve relative to the most competitive structural model of Pnnm for the predicted $\mathrm{P}_{2} / \mathrm{m}$ phase is presented in Figure $2 \mathrm{~b}$. It can be seen that the 

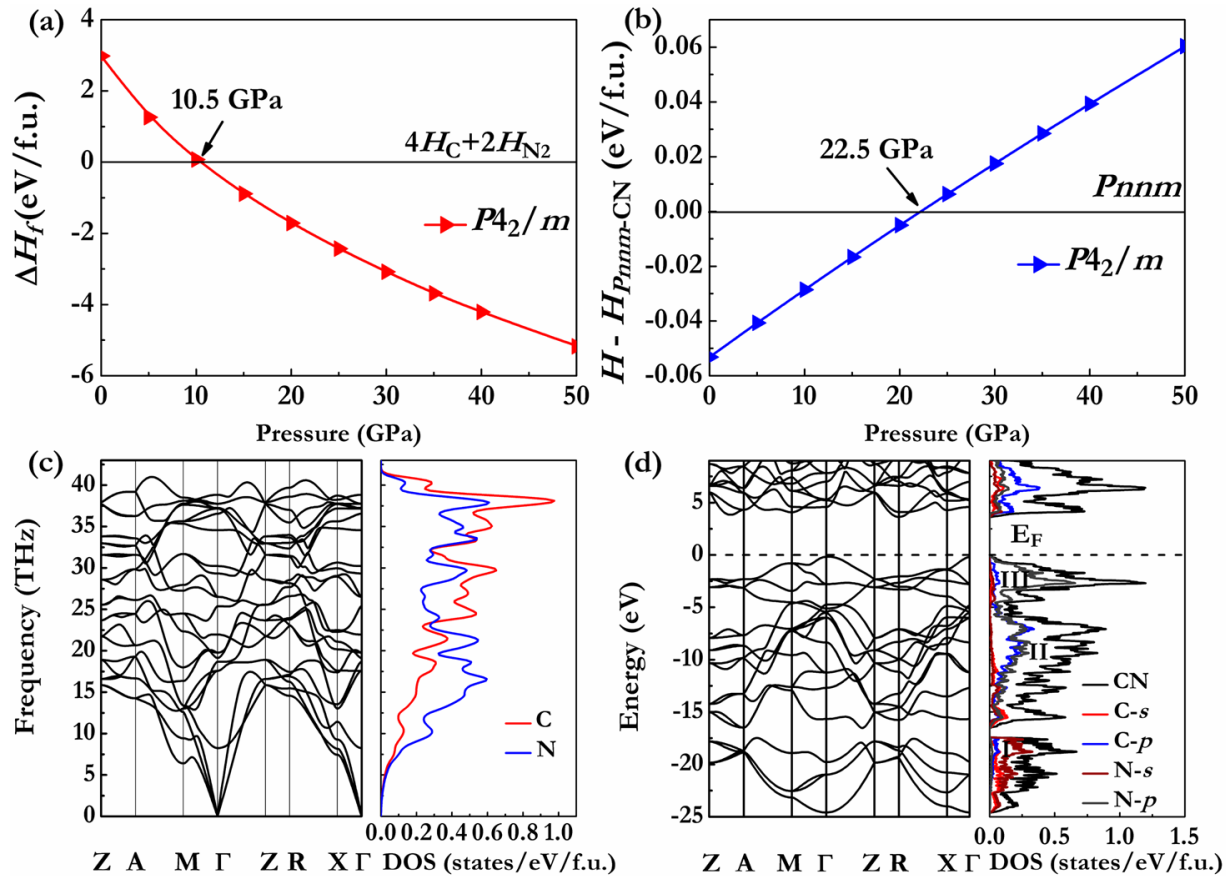

Figure 2. Formation enthalpies of $P 4_{2} / m-\mathrm{CN}$ relative to graphite and nitrogen as a function of pressure (a), enthalpy differences for $P 4_{2} / m$ - $\mathrm{CN}$ relative to $P n n m-\mathrm{CN}$ as a function of pressure (b), phonon dispersion curves of $P 4_{2} / \mathrm{m}$-CN at $0 \mathrm{GPa}$ (c), and band structure and density of states (DOS) of $P 4_{2} / m-\mathrm{CN}$ at $0 \mathrm{GPa}(\mathrm{d})$.

Table 1. Calculated Elastic Constants $C_{i j}$, Bulk Modulus B, Shear Modulus G, and Young's Modulus E (in units of GPa) of P4 $/$ $m$-CN. Also Shown are $G / B$ Ratio and Poisson's Ratio $v$

\begin{tabular}{|c|c|c|c|c|c|c|c|c|c|c|c|c|c|c|}
\hline phase & $C_{11}$ & $C_{22}$ & $C_{33}$ & $C_{44}$ & $C_{55}$ & $C_{66}$ & $C_{12}$ & $C_{13}$ & $C_{23}$ & B & G & E & $G / B$ & $v$ \\
\hline $\mathrm{P}_{2} / \mathrm{m}-\mathrm{CN}$ & 596 & & 1156 & 370 & & 326 & 172 & 116 & & 341 & 328 & 745 & 0.96 & 0.136 \\
\hline Pnnm-CN & 518 & 767 & 1227 & 534 & 277 & 379 & 203 & 83 & 201 & 369 & 351 & 799 & 0.95 & 0.139 \\
\hline$c g-\mathrm{CN}^{a}$ & 445 & & & 230 & & & 280 & & & 335 & & & & \\
\hline$\beta-C_{3} \mathrm{~N}_{4}{ }^{b}$ & 922 & & 1053 & 330 & & 374 & 173 & 116 & & 412 & 370 & 855 & 0.9 & 0.15 \\
\hline$b c t-\mathrm{CN}_{2}{ }^{b}$ & 836 & & 1269 & 397 & & 313 & 150 & 120 & & 407 & 386 & 879 & 0.95 & 0.14 \\
\hline$c-\mathrm{BN}^{c}$ & 820 & & & 480 & & & 190 & & & 400 & & & & \\
\hline diamond $^{d}$ & 1076 & & & 577 & & & 125 & & & 442 & & & & \\
\hline
\end{tabular}

predicted tetragonal $P 4_{2} / m$ structure is much more stable than the Pnnm phase below a pressure of $22.5 \mathrm{GPa}$, above which the Pnnm structure becomes energetically favorable in the studied pressure ranges. Therefore, present calculations give direct evidence that the predicted $P 4_{2} / m-C N$ is the most energetically preferable structure in the known structures at ambient pressure and can be synthesized at readily attainable pressures. Further experimental work is thus strongly recommended.

We have plotted the band structure an total and partial density of state (DOS) of $P 4_{2} / m-C N$ at $0 \mathrm{GPa}$ in Figure $2 \mathrm{~d}$. This tetragonal phase is an insulator with an indirect band gap of $3.8 \mathrm{eV}$, suggesting that electronic excitation is more difficult to achieve even if the optical energy matches the value of band gaps. Because DFT calculations usually lead to its underestimation, the actual band gap is expected to be much larger. From inspection of its partial DOS curves in Figure 2d, the valence band region can be divided into three groups of states depicted as I, II, and III. Part I originated from C-s states mixed with contributions of $\mathrm{N}$-s states. Part II and part III are characterized by a mixture of $\mathrm{C}-\mathrm{p}$ and $\mathrm{N}-\mathrm{p}$ states. The strong $\mathrm{C}-\mathrm{N}$ covalent bonding nature in $\mathrm{P}_{2} / \mathrm{m}-\mathrm{CN}$ is revealed by the $-25 \mathrm{eV}$ to Fermi level $\left(E_{\mathrm{F}}\right)$ because of the similar partial DOS profiles of C-s, C-p, N-s, and N-p states. Moreover, another C$\mathrm{C}$ covalent bonding nature can be seen from their partial DOS profiles, although it is not as strong as $\mathrm{C}-\mathrm{N}$ bonds. In the conduction band region of DOS, the peaks are mainly contributed by $\mathrm{C}-\mathrm{p}$ states. We found through Bader charge analysis that the charge transfer from $\mathrm{C}$ to $\mathrm{N}$ atom is $0.95 \mathrm{e}$ in one f.u., indicating the ionicity of $\mathrm{C}-\mathrm{N}$ bonds. Therefore, the chemical bonding between $\mathrm{C}$ and $\mathrm{N}$ atoms in $P 4_{2} / \mathrm{m}-\mathrm{CN}$ is a complex mixture of covalent and ionic characteristics.

To study the mechanical properties of $P 4_{2} / m-C N$, the elastic constants are calculated by using the strain-stress method. For tetragonal $P 4_{2} / m-C N$, there are six independent elastic constants, which were calculated and are listed in Table 1. The mechanical stabilities of $\mathrm{P}_{2} / \mathrm{m}-\mathrm{CN}$ satisfy the BornHuang criteria ${ }^{42}$ for a tetragonal crystal $\left[C_{11}>0, C_{33}>0, C_{44}>\right.$ $0, C_{66}>0, C_{11}-C_{12}>0, C_{11}+C_{33}-2 C_{13}>0,2\left(C_{11}+C_{12}\right)+$ $\left.C_{33}+4 C_{13}>0\right]$, indicating that it is mechanically stable at ambient pressure. From Table 1, we find unusually high incompressibility along $c$-direction for $\mathrm{P}_{2} / \mathrm{m}-\mathrm{CN}$, as demonstrated by the extremely large $C_{33}$ value $(1156 \mathrm{GPa})$, which is slightly larger than the experimental data of diamond $\left(C_{33}\right.$ : $1076 \mathrm{GPa}){ }^{43}$ This value is comparable to those of known 

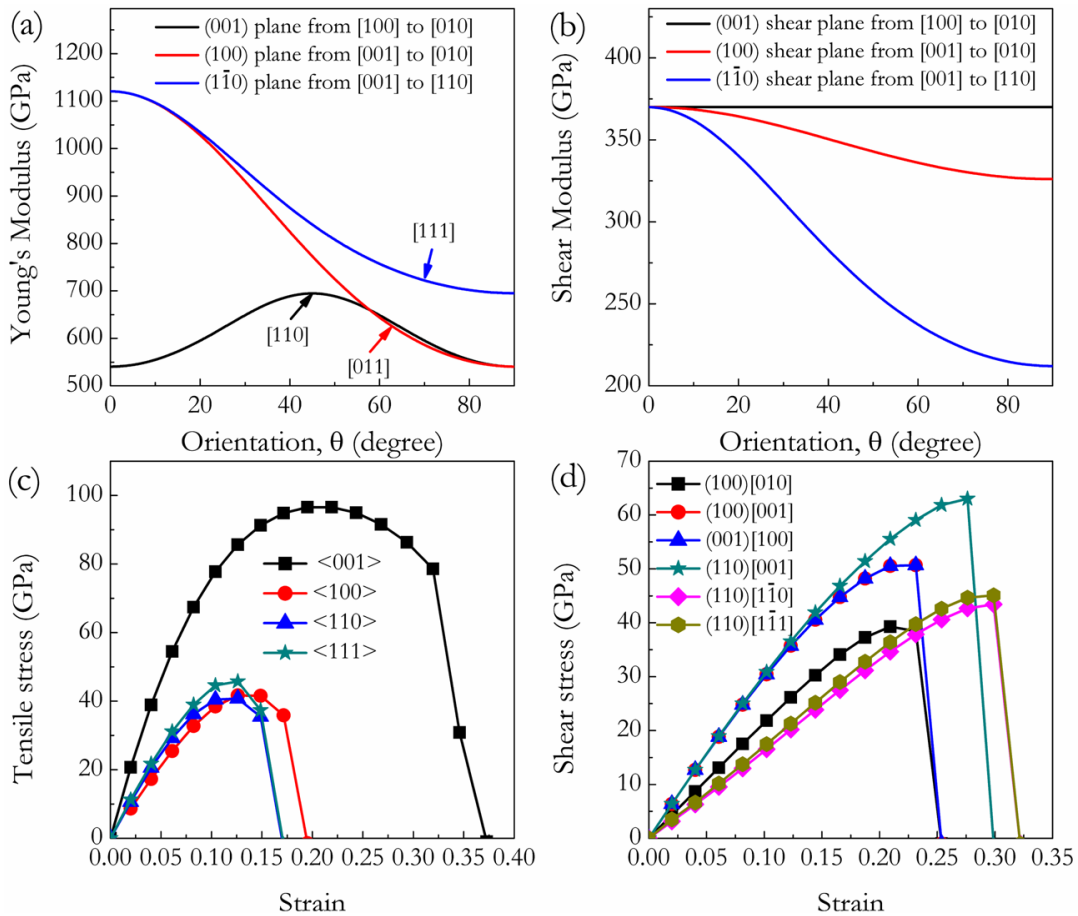

Figure 3. Orientation dependence of the Young's modulus in $P 4_{2} / m-\mathrm{CN}(\mathrm{a})$, orientation dependence of the shear modulus in $P 4_{2} / \mathrm{m}$ - $\mathrm{CN}(\mathrm{b})$, and calculated stress-strain relations of $P 4_{2} / m-C N$ in various tensile (c) and shear (d) directions.

potential superhard materials $\left(\beta-\mathrm{C}_{3} \mathrm{~N}_{4}(1053 \mathrm{GPa}){ }^{12} b c t-\mathrm{C}_{4}\right.$ $(1189.9 \mathrm{GPa}){ }^{44}$ and $Z$-carbon $\left.(1184.5 \mathrm{GPa})^{44}\right)$ suggesting that the $c$-axial direction of $P 4_{2} / \mathrm{m}$-CN is very stiff. The polycrystalline bulk modulus $B$, shear modulus $G$, Young's modulus $E$, Poisson's ratio $v$, and $G / B$ of $P 4_{2} / m-C N$ are also shown in Table 1. Because the hardness is deduced from the size of the indentation after deformation, a hard material typically requires a high bulk modulus to support the volume decrease created by the applied pressure and a low Poisson's ratio $(\nu)$ or high shear modulus (so that the material will not deform in a direction different from that of the applied load). The calculated bulk modulus of $P 4_{2} / \mathrm{m}$-CN is comparable to those of $b c t-\mathrm{C}_{4}(403.4$ $\mathrm{GPa}),{ }^{44} b c t-\mathrm{CN}_{2}(392 \mathrm{GPa}){ }^{12} \alpha-\mathrm{C}_{3} \mathrm{~N}_{2}(380 \mathrm{GPa}),{ }^{11} \beta-\mathrm{C}_{3} \mathrm{~N}_{2}$ $(343 \mathrm{GPa}),{ }^{11} c$-BN $(400 \mathrm{GPa})^{45}$ and $\beta-\mathrm{C}_{11} \mathrm{~N}_{4}(367 \mathrm{GPa}){ }^{46}$ confirming that $P 4_{2} / m-C N$ is a highly incompressible material. According to Teter, ${ }^{47}$ the shear modulus is a significantly better qualitative predictor of hardness than the bulk modulus, governing the indentation hardness. The shear modulus of $\mathrm{P}_{2} / \mathrm{m}$-CN is $328 \mathrm{GPa}$ and it is expected to withstand shear strain to a large extent.

To understand the elastic deformation of $P 4_{2} / m-C N$, it is important to know the orientation dependences of the Young's modulus $E$ and shear modulus $G$, as discussed in previous works. $^{48}$ For the $P 4_{2} / m-\mathrm{CN}$, the Young's modulus $E$ is described by the following equation:

$$
\begin{aligned}
& E^{-1}=s_{11}\left(\alpha^{4}+\beta^{4}\right)+s_{33} \gamma^{4}+2 s_{12} \alpha^{2} \beta^{2} \\
& \quad+2 s_{13}\left(\beta^{2} \gamma^{2}+\alpha^{2} \gamma^{2}\right)+s_{44}\left(\beta^{2} \gamma^{2}+\alpha^{2} \gamma^{2}\right)+s_{66} \alpha^{2} \beta^{2}
\end{aligned}
$$

where $\alpha, \beta$, and $\gamma$ are the direction cosines which determine the angles between the $a$-, $b$-, and $c$-axis of a crystal and a given direction $[u v w] . s_{11}, s_{33}, s_{44}, s_{66}, s_{12}$, and $s_{13}$ are the elastic compliance constants which are given by Kelly et al. ${ }^{49}$ Similarly, the orientation dependence of the shear modulus for arbitrary shear plane $(h k l)$ and shear directions $[u v w]$ is given by

$$
\begin{aligned}
& G^{-1}=4 s_{11}\left(\alpha_{1}^{2} \alpha_{2}^{2}+\beta_{1}^{2} \beta_{2}^{2}\right)+4 s_{33} \gamma_{1}^{2} \gamma_{2}^{2}+8 s_{12} \alpha_{1} \alpha_{2} \beta_{1} \beta_{2} \\
& +s_{66}\left(\alpha_{1} \beta_{2}+\alpha_{2} \beta_{1}\right)^{2}+8 s_{13}\left(\beta_{1} \beta_{2} \gamma_{1} \gamma_{2}+\alpha_{1} \alpha_{2} \gamma_{1} \gamma_{2}\right) \\
& +s_{44}\left[\left(\beta_{1} \gamma_{2}+\beta_{2} \gamma_{1}\right)^{2}+\left(\alpha_{1} \gamma_{2}+\alpha_{2} \gamma_{1}\right)^{2}\right]
\end{aligned}
$$

where $\left(\alpha_{1}, \beta_{1}, \gamma_{1}\right)$ and $\left(\alpha_{2}, \beta_{2}, \gamma_{2}\right)$ are the direction cosines of the $[u v w]$ and $[H K L]$ directions and the $[H K L]$ denotes the vector normal to the $(h k l)$ shear plane. The results of these calculations for the $P 4_{2} / \mathrm{m}-\mathrm{CN}$ are summarized in Figure 3a for $E$ and Figure $3 b$ for $G$. From Figure $3 \mathrm{a}$, it can be seen that (1) the maximum and minimum values of $P 4_{2} / \mathrm{m}-\mathrm{CN}$ are along the [001] $\left(E_{[001]}=1121 \mathrm{GPa}\right)$ and $[100]\left(E_{[100]}=540 \mathrm{GPa}\right)$ directions, (2) $E_{\max } / E_{\min }=2.08$, and (3) the variations of the Young's moduli along different directions decrease in the following sequence: $E_{[001]}>E_{[111]}>E_{[110]}>E_{[011]}>E_{[100]}$. Therefore, the Young's moduli $E$ for different tensile axes in Figure 3a suggest that the $P 4_{2} / \mathrm{m}$-CN possesses a high degree of anisotropy. As plotted in Figure $3 b$, the orientation dependence of the shear modulus $G$ of the $P 4_{2} / m-C N$ was also calculated for shear on (001), (100), and (110). The shear modulus of

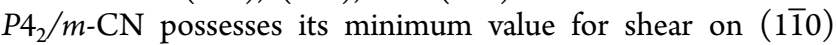
$[110]\left(E_{(1 \overline{1} 0)[110]}=212 \mathrm{GPa}\right)$ and its maximum value for shear on (001), where it does not depend on shear direction, and on $(100)[001]\left(E_{(100)[001]}=370 \mathrm{GPa}\right)$.

In view of the large elastic moduli of $P 4_{2} / m-C N$, the hardness calculations are of great interest. Previous studies ${ }^{50,51}$ have demonstrated that ultimate hardness of a material can be assessed from its ideal strength and bonding nature, which also appears to correlate with the onset of dislocation formation in an ideal, defect-free crystal. Therefore, we next discuss the ideal strength from the elastic regime to the limit of its structural stability and understand the underlying bond-responding processes under large strains. Figure $3 \mathrm{c}, \mathrm{d}$ shows the stressstrain curves calculated for $P 4_{2} / m-C N$. The ideal tensile strength of $P 4_{2} / \mathrm{m}-\mathrm{CN}$ along the $\langle 001\rangle$ direction is about 

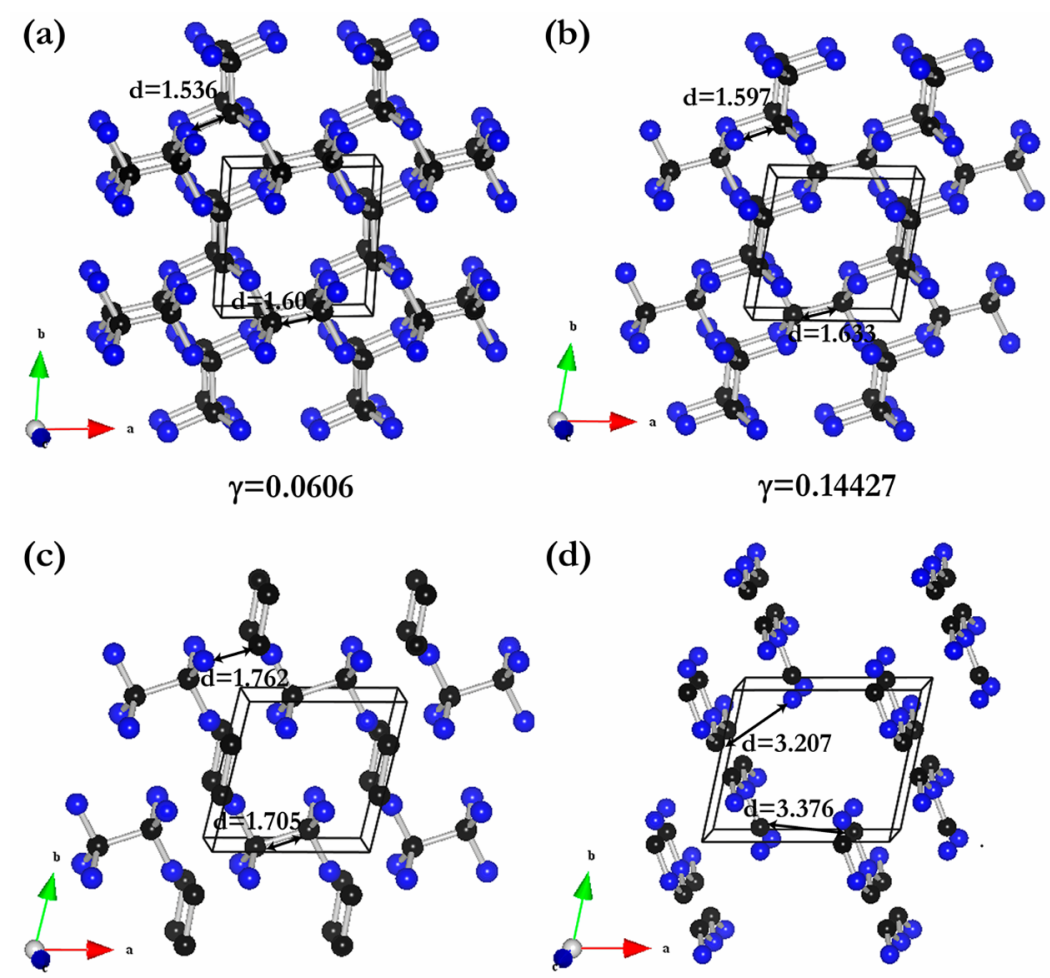

$\gamma=0.2313$

$\gamma=0.2536$

Figure 4. Atomic bond structures $P 4_{2} / \mathrm{m}$-CN before and after lattice instability. The arrowheads indicate the instability of $\mathrm{C}-\mathrm{N}$ and $\mathrm{C}-\mathrm{C}$ bonds upon shear.
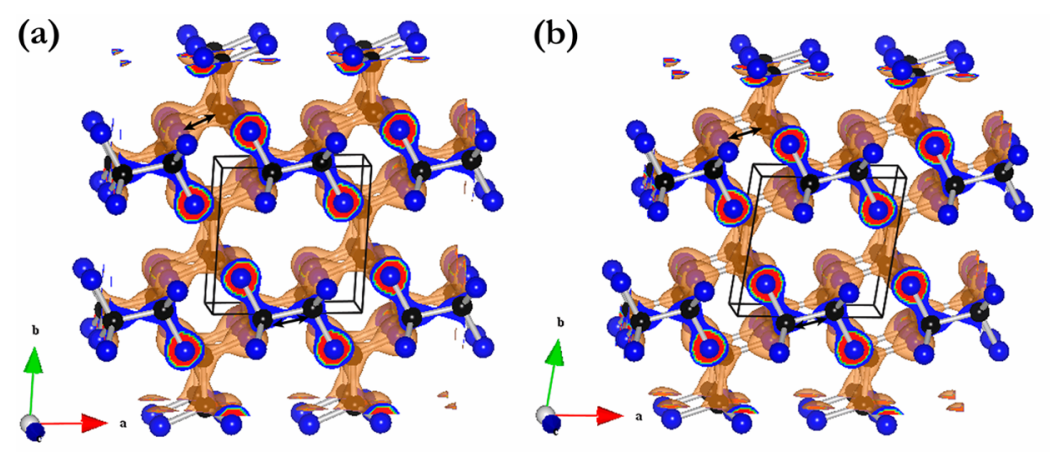

$$
\gamma=0.0606
$$

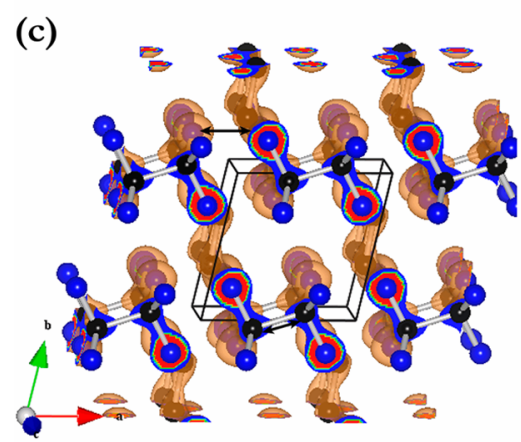

$\gamma=0.2313$

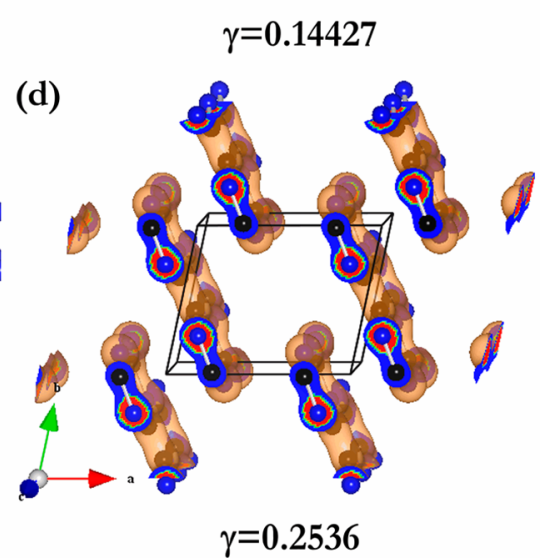

Figure 5. Corresponding 3D charge density distributions of $\mathrm{P}_{2} / \mathrm{m}-\mathrm{CN}$ before and after lattice instability.

96.6 GPa, which is close to those of $h c p-\mathrm{ReB}_{2}\left(\sigma_{\langle 0001\rangle}=93.2\right.$ $\mathrm{GPa})^{52}$ and $b c t-\mathrm{CN}_{2}\left(\sigma_{\langle 001\rangle}=117.1 \mathrm{GPa}\right) .{ }^{12}$ The anisotropy ratio of tensile strengths of $\sigma_{\langle 110\rangle}(40.8 \mathrm{GPa}): \sigma_{\langle 100\rangle}(41.5 \mathrm{GPa})$ : $\sigma_{\langle 111\rangle}(45.8 \mathrm{GPa}): \sigma_{\langle 001\rangle}(96.6 \mathrm{GPa})=1: 1.02: 1.12: 2.37$ indicates that it is nearly isotropic within the (001) plane, similar to the well-known elastic isotropy in the tetragonal 
phase in this plane. The calculations of ideal shear strength upon large strains can examine the anisotropy of shear deformation where plastic deformation proceeds irreversibly on the atomic scale. First, the values of the ideal shear strengths of the $P 4_{2} / \mathrm{m}$-CN along the (001) [100] deformation path is about $50.7 \mathrm{GPa}$ in Figure 3c; this value is close to that of $c$-BN $(58.3 \mathrm{GPa})$ but larger than that of $\operatorname{ReB}_{2}(34.4 \mathrm{GPa}) .{ }^{52}$ Second, the weakest peak tensile stress occurs in the [110] direction as shown in Figure 3c, which indicates that under tensile loadings $P 4_{2} / \mathrm{m}$-CN would first cleave in the (110) plane. The critical shear stress in the $P 4_{2} / m-C N$ was then calculated by applying $[001],[1 \overline{1} 0]$, and $[1 \overline{1} 1]$ shear deformations in the (110) easy cleavage plane perpendicular to the weakest tensile direction, as shown in Figure 3d. The lowest shear strength appears in the (110) [1 $1 \overline{1} 0]$ direction with a peak shear stress of $43.4 \mathrm{GPa}$. Third, the shear strength in the (100)[010] and (100)[001] directions are also shown in Figure $3 \mathrm{~d}$ for comparison. As indicated in Figure $3 \mathrm{~d}$, the lowest peak shear stress occurs in the (100)[010] direction with $40 \mathrm{GPa}$, which is lower than the lowest tensile strengths. This means the failure mode in $\mathrm{P}_{2} / \mathrm{m}$ $\mathrm{CN}$ is dominated by the shear type, which is similar to that in $\beta-\mathrm{C}_{3} \mathrm{~N}_{4}$. To shed light on the origin of the deformation mechanism in the (100)[010] direction, the bond arrangements and corresponding charge density distributions of $\mathrm{P4}_{2} /$ $m-\mathrm{CN}$ before and after the shear instability were analyzed and plotted in Figures 4 and 5. At selected increasing shear strains, both $\mathrm{C}-\mathrm{N}$ bonds in the helical tunnels and $\mathrm{C}-\mathrm{C}$ bonds connected to these helical tunnels become unstable and break at the critical shear strains. The $\mathrm{C}-\mathrm{N}$ bonds indicated by arrows in the helical tunnels are stretched under increasing shear strains and break at $\gamma=0.2313$, which limits the achievable strength of $P 4_{2} / \mathrm{m}$-CN. This means that this shearinduced structural transformation first occurs at a strain of 0.2313 through the collapse of 3D helical tunnels by simultaneously breaking the $\mathrm{C}-\mathrm{N}$ bonds. The breaking of $\mathrm{C}-\mathrm{C}$ bonds has been found to occur at $\gamma=0.2536$ with longer bonds, resulting in the formation of coplanar graphite-like $\mathrm{C}-\mathrm{N}$ layers in $P 4_{2} / m-C N$. In addition, this could be further confirmed by the charge density of $P 4_{2} / m-C N$ at selected increasing shear strains in Figure 5. The instability of $\mathrm{C}-\mathrm{N}$ and $\mathrm{C}-\mathrm{C}$ bonds under shear deformation for $\mathrm{P} 4_{2} / \mathrm{m}-\mathrm{CN}$ can be attributed to a local transformation of $\mathrm{sp}^{3}$ to $\mathrm{sp}^{2}$ upon the shear.

\section{CONCLUSIONS}

In summary, a new tetragonal $P 4_{2} / m-C N$ has been explored by first-principles calculations and crystal structure prediction techniques. When compared with previously reported $\mathrm{CN}$ phases with 1:1 stoichiometry, the $P 4_{2} / \mathrm{m}-\mathrm{CN}$ phase is the most stable at ambient pressure. Phonon dispersion calculations demonstrate that $\mathrm{P4}_{2} / \mathrm{m}$-CN is dynamically stable at ambient conditions. The crystal orientation dependences of the Young's and shear moduli have been systematically studied for this $\mathrm{P4}_{2} /$ $m$ phase. The predicted elastic moduli, tensile strength, and shear strength indicate that it is ultracompressible and potentially superhard, originating from the strong $\mathrm{C}-\mathrm{N}$ and $\mathrm{C}-\mathrm{C}$ covalent bonding networks. Thus, we hope that additional high-pressure experiments can be done on the synthesis of the superhard material for applications.

\section{AUTHOR INFORMATION}

\section{Corresponding Authors}

*M.Z.: phone, +86 917 3364258; e-mail, zhmgbj@126.com.

*Q.W.: phone, +86 29 88469165; e-mail, weiaqun@163.com.

\section{Notes}

The authors declare no competing financial interest.

\section{ACKNOWLEDGMENTS}

This work was supported by the National Natural Science Foundation of China (Grant 11204007), the Natural Science Basic Research plan in Shaanxi Province of China (Grant 2012JQ1005, 2013JQ1007), the Fundamental Research Funds for the Central Universities, and the Education Committee Natural Science Foundation in Shaanxi Province of China (Grant 2013JK0638).

\section{REFERENCES}

(1) Léger, J. M.; Haines, J. The Search for Superhard Materials. Endeavour 1997, 21, 121-124.

(2) Liu, A. Y.; Cohen, M. L. Prediction of New Low Compressibility Solids. Science 1989, 245, 841-842.

(3) Liu, A. Y.; Cohen, M. L. Structural Properties and Electronic Structure of Low-compressibility Materials: $\beta-\mathrm{Si}_{3} \mathrm{~N}_{4}$ and Hypothetical $\beta$ - $\mathrm{C}_{3} \mathrm{~N}_{4}$. Phys. Rev. B 1990, 41, 10727-10734.

(4) Côté, M.; Cohen, M. L. Carbon Nitride Compounds with 1:1 Stoichiometry. Phys. Rev. B 1997, 55, 5684-5688.

(5) Guo, L. P.; Chen, Y.; Wang, E. G.; Li, L.; Zhao, Z. X. Identification of a New Tetragonal CN Phase. J. Cryst. Growth 1997, $178,639-644$.

(6) Hales, J.; Barnard, A. S. Thermodynamic Stability and Electronic Structure of Small Carbon Nitride Nanotubes. J. Phys.: Condens. Matter 2009, 21, 144203.

(7) Kim, E.; Chen, C.; Kohler, T.; Elstner, M.; Frauenheim, T. Tetragonal Crystalline Carbon Nitrides: Theoretical Predictions. Phys. Rev. Lett. 2001, 86, 652-655.

(8) Kim, E.; Chen, C. Stability of Tetragonal Crystalline Carbon Nitrides: The Nitrogen Content Dependence. Phys. Lett. A 2001, 282, 415-420.

(9) Hart, J. N.; Claeyssens, F.; Allan, N. L.; May, P. W. Carbon Nitride: $\mathrm{Ab}$ Initio Investigation of Carbon-rich Phases. Phys. Rev. B 2009, 80, 174111.

(10) Sandré, E.; Pickard, C. J.; Colliex, C. What are the Possible Structures for $\mathrm{CN}_{x}$ Compounds? The Example of $\mathrm{C}_{3} \mathrm{~N}$. Chem. Phys. Lett. 2000, 325, 53-60.

(11) Tian, F. B.; Wang, J. H.; He, Z.; Ma, Y. M.; Wang, L. C.; Cui, T.; Chen, C. B.; Liu, B. B.; Zou, G. T. Superhard Semiconducting $\mathrm{C}_{3} \mathrm{~N}_{2}$ Compounds Predicted via First-principles Calculations. Phys. Rev. B 2008, 78, 235431.

(12) Li, Q.; Liu, H. Y.; Zhou, D.; Zheng, W. T.; Wu, Z. J.; Ma, Y. M. A Novel Low Compressible and Superhard Carbon Nitride: Bodycentered Tetragonal $\mathrm{CN}_{2}$. Phys. Chem. Chem. Phys. 2012, 14, 1308113087.

(13) Sjöström, H.; Stafström, S.; Boman, M.; Sundgren, J.-E. Superhard and Elastic Carbon Nitride Thin Films Having Fullerenelike Microstructure. Phys. Rev. Lett. 1995, 75, 1336-1339.

(14) Sekine, T.; Kanda, H.; Bando, Y.; Yokoyama, M.; Hojou, K. A Graphitic Carbon Nitride. J. Mater. Sci. Lett. 1990, 9, 1376-1378.

(15) Stevens, A. J.; Koga, T.; Agee, C. B.; Aziz, M. J.; Lieber, C. M. Stability of Carbon Nitride Materials at High Pressure and Temperature. J. Am. Chem. Soc. 1996, 118, 10900-10901.

(16) Stevens, A. J.; Agee, C. B.; Lieber, C. M. High-pressure Chemistry of Carbon Nitride Materials. Mater. Res. Soc. Symp. Proc. 1998, 499, 309.

(17) Chen, Y.; Guo, L.; Chen, F.; Wang, E. G. Synthesis and Characterization of $\mathrm{C}_{3} \mathrm{~N}_{4}$ Crystalline Films on Silicon. J. Phys.: Condens. Matter 1996, 8, L685.

(18) Wang, E. G. Research on Carbon Nitride. Prog. Mater. Sci. 1997, 41, 241-298.

(19) Muhl, S.; Méndez, J. M. A Review of the Preparation of Carbon Nitride Films. Diamond Relat. Mater. 1999, 8, 1809-1830.

(20) Khazaei, M.; Tripathi, M. N.; Kawazoe, Y. First-principles Simulation of Cyanogen under High Pressure: Formation of 
Paracyanogen and an Insulating Carbon Nitride Solid. Phys. Rev. B 2011, 83, 134111.

(21) Román-Pérez, G.; Zamora, F.; Soler, J. M. Hollow $\mathrm{C}_{3} \mathrm{~N}_{4}$ Nanoclusters from First Principles. Phys. Rev. B 2010, 82, 195405.

(22) Thomas, A.; Fischer, A.; Goettman, F.; Antonietti, M.; Müller, J.-O.; Schlögl, R.; Carlsson, J. M. Graphitic Carbon Nitride Materials: Variation of Structure and Morphology and Their Use as Metal-free Catalysts. J. Mater. Chem. 2008, 18, 4893-4908.

(23) Wang, X. L.; Bao, K.; Tian, F. B.; Meng, X.; Chen, C. B.; Dong, B. W.; Li, D.; Liu, B. B.; Cui, T. Cubic Gauche-CN: A Superhard Metallic Compound Predicted via First-principles Calculations. J. Chem. Phys. 2010, 133, 044512.

(24) Li, Q.; Ma, Y. M.; Oganov, A. R.; Wang, H.; Xu, Y.; Cui, T.; Mao, H. K.; Zou, G. T. Superhard Monoclinic Polymorph of Carbon. Phys. Rev. Lett. 2009, 102, 175506.

(25) Boulfelfel, S. E.; Oganov, A. R; Leoni, S. Understanding the Nature of "Superhard Graphite". Sci. Rep. 2012, 2, 471.

(26) Wang, Y.; Panzik, J. E.; Kiefer, B.; Lee, K. K. M. Crystal Structure of Graphite under Room-temperature Compression and Decompression. Sci. Rep. 2012, 2, 520.

(27) Wang, X. L. Polymorphic Phases of $s p^{3}$-hybridized Superhard CN. J. Chem. Phys. 2012, 137, 184506.

(28) Wang, Y. C.; Lv, J.; Zhu, L.; Ma, Y. M. Crystal Structure Prediction via Particle Swarm Optimization. Phys. Rev. B 2010, 82, 094116.

(29) Nishio-Hamane, D.; Zhang, M. G.; Yagi, T.; Ma, Y. M. Highpressure and High-temperature Phase Transitions in $\mathrm{FeTiO}_{3}$ and a New Dense $\mathrm{FeTi}_{3} \mathrm{O}_{7}$ Structure. Am. Mineral. 2012, 97, 568-572.

(30) Lv, J.; Wang, Y. C.; Zhu, L.; Ma, Y. M. Predicted Novel HighPressure Phases of Lithium. Phys. Rev. Lett. 2011, 106, 015503.

(31) Zhu, L.; Wang, H.; Wang, Y. C.; Lv, J.; Ma, Y. M.; Cui, Q. L.; Ma, Y. M.; Zou, G. T. Substitutional Alloy of Bi and Te at High Pressure. Phys. Rev. Lett. 2011, 106, 145501.

(32) Wang, Y. C.; Lv, J.; Zhu, L.; Ma, Y. M. CALYPSO: A Method for Crystal Structure Prediction. Comput. Phys. Commun. 2012, 183, 2063-2070.

(33) Kresse, G.; Furthmüller, J. Efficient Iterative Schemes for Ab Initio Total-Energy Calculations Using a Plane-Wave Basis Set. Phys. Rev. B 1996, 54, 11169-11186.

(34) Perdew, J. P.; Burke, K.; Ernzerhof, M. Generalized Gradient Approximation Made Simple. Phys. Rev. Lett. 1996, 77, 3865-3868.

(35) Kresse, G.; Joubert, D. From Ultrasoft Pseudopotentials to the Projector Augmented-Wave Method. Phys. Rev. B 1999, 59, 17581775 .

(36) Monkhorst, H. J.; Pack, J. D. Special Points for Brillouin-Zone Integrations. Phys. Rev. B 1976, 13, 5188-5192.

(37) Milman, V.; Warren, M. C. Elasticity of Hexagonal BeO. J. Phys.: Condens. Matter 2001, 13, 241.

(38) Hill, R. The Elastic Behaviour of a Crystalline Aggregate. Proc. Phys. Soc., London, Sect. A 1952, A65, 349-354.

(39) Roundy, D.; Krenn, C. R.; Cohen, M. L.; Morris, J. W., Jr. The Ideal Strength of Tungsten. Philos. Mag. 2001, A81, 1725-1747.

(40) Togo, A.; Oba, F.; Tanaka, I. First-principles Calculations of the Ferroelastic Transition between Rutile-type and $\mathrm{CaCl}_{2}$-type $\mathrm{SiO}_{2}$ at High Pressures. Phys. Rev. B 2008, 78, 134106.

(41) Venables, J. A.; English, C. A. Electron Diffraction and the Structure of $\alpha-\mathrm{N}_{2}$. Acta Crystallogr., Sect. B: Struct. Crystallogr. Cryst. Chem. 1974, 30, 929-935.

(42) Born, M.; Huang, K. Dynamical Theory of Crystal Lattices; Clarendon Press, Oxford, U.K., 1956.

(43) Grimsditch, M. H.; Ramdas, A. K. Brillouin Scattering in Diamond. Phys. Rev. B 1975, 11, 3139-3148.

(44) Li, Z. P.; Gao, F. M.; Xu, Z. M. Strength, Hardness, and Lattice Vibrations of Z-carbon and W-carbon: First-principles Calculations. Phys. Rev. B 2012, 85, 144115.

(45) Grimsditch, M.; Zouboulis, E. S.; Polian, A. Elastic Constants of Boron Nitride. J. Appl. Phys. 1994, 76, 832.
(46) Mattesini, M.; Matar, S. Density-functional Theory Investigation of Hardness, Stability, and Electron-energy-loss Spectra of Carbon Nitrides with $\mathrm{C}_{11} \mathrm{~N}_{4}$ Stoichiometry. Phys. Rev. B 2002, 65, 075110.

(47) Teter, D. M. Computational Alchemy: The Search for New Superhard Materials. Mater. Res. Soc. Bull. 1998, 23, 22-27.

(48) He, Y.; Schwarz, R. B.; Migliori, A. Elastic Constants of Single Crystal $\gamma$-TiAl. J. Mater. Res. 1995, 10, 1187-1195.

(49) Kelly, A.; MacMillan, N. H. Strong Solids; Oxford University Press: Oxford, U.K., 1986.

(50) Zhang, Y.; Sun, H.; Chen, C. F. Superhard Cubic $\mathrm{BC}_{2} \mathrm{~N}$ Compared to Diamond. Phys. Rev. Lett. 2004, 93, 195504.

(51) Roundy, D.; Krenn, C. R.; Cohen, M. L.; Morris, J. W., Jr. Ideal Shear Strengths of fcc Aluminum and Copper. Phys. Rev. Lett. 1999, $82,2713-2716$.

(52) Zhang, R. F.; Veprek, S.; Argon, A. S. Mechanical and Electronic Properties of Hard Rhenium Diboride of Low Elastic Compressibility Studied by First-principles Calculation. Appl. Phys. Lett. 2007, 91, 201914. 